\title{
IgA autoantibodies to gliadin nonapeptides, tissue transglutaminase and epidermal transglutaminase are associated, but unrelated to neutrophil elastase expression in lesional skin in human dermatitis herpetiformis
}

\author{
Justyna Gornowicz-Porowskaํ, Agnieszka Seraszek-Jaros², Elżbieta Kaczmarek², Marian Dmochowski ${ }^{1}$, \\ Monika Bowszyc-Dmochowska ${ }^{1}$
}

${ }^{1}$ Cutaneous Histopathology and Immunopathology Section, Department of Dermatology, Poznan University of Medical Sciences, Poland Head: Assist. Prof. Monika Bowszyc-Dmochowska MD, PhD

2Department of Bioinformatics and Computational Biology, Poznan University of Medical Sciences, Poland Head: Prof. Elżbieta Kaczmarek

Postep Derm Alergol 2012; XXIX, 4: 233-239

DOI: $10.5114 /$ pdia.2012.30461

\begin{abstract}
Introduction: In dermatitis herpetiformis (DH), activation of neutrophils via IgA Fc receptors (e.g. CD89) and immunocomplexes containing IgA1 may lead to the release of proteolytic enzymes, mainly neutrophil elastase (NE), destruction of the dermal-epidermal junction and blister formation.

Aim: To analyze whether there are associations between levels of circulating IgA autoantibodies to epidermal (eTG) and tissue (tTG) transglutaminases, nonapeptides of gliadin (npG) and the intensity of cutaneous NE expression in patients with $\mathrm{DH}$.

Material and methods: Altogether, 31 patients with DH were studied. The levels of serum IgA autoantibodies to eTG, tTG and npG were evaluated with ELISAs. The intensity of NE expression was estimated with immunohistochemistry using NE monoclonal antibody and quantitative digital morphometry. Statistical analysis was done using Spearman's rank correlation coefficient.

Results: There were no statistically significant correlations between the intensity of cutaneous NE expression and the levels of serum IgA autoantibodies to eTG, tTG, npG in patients with DH. There were statistically significant correlations between the levels of IgA autoantibodies to eTG, tTG, npG.

Conclusions: It seems that in human DH the activation of neutrophils, judged by the NE expression, is unrelated to the levels of serum IgA autoantibodies to eTG/tTG/npG, but all those autoantibodies in $\mathrm{DH}$ are produced in a coordinated way.
\end{abstract}

Key words: dermatitis herpetiformis, neutrophil elastase, transglutaminases, gliadin.

\section{Introduction}

Dermatitis herpetiformis (DH) was first described by Louis Adolphus Duhring from Philadelphia, USA in 1884 [1], however up to now the precise molecular mechanism of blister formation in $\mathrm{DH}$ remains unclear. Indeed, the induction of autoimmune response in $\mathrm{DH}$, the subepidermal autoimmune blistering dermatosis, is a complex process resulting from interplay of both genetic and environmental factors. The main autoantigens in $\mathrm{DH}$ are plau- sibly recognized by principally IgA1 autoantibodies [2, 3] and involve epidermal transglutaminase (eTG, TG3) and closely related tissue transglutaminase (tTG, TG2) [4, 5]. Nevertheless, current data indicated that nonapeptides of gliadin (npG) may be considered in the pathomechanism/diagnosis of DH [6]. The stereotypical cutaneous lesion is characterized microscopically by the neutrophilrich infiltrate at the dermal-epidermal junction (DEJ), which often localizes to the papillary tips $[7,8]$. Thus, 
the activation of neutrophils may be essential for development of DH. Recently obtained data [2, 9] suggested the involvement of an impaired human IgA Fc receptors (FcRs) regulatory system that may cause the activation of neutrophils with subsequent secretion of various proteases, including neutrophil elastase (NE), responsible for the DEJ destruction. It was demonstrated that circulating neutrophils from DH patients show evidence of partial priming including the increased function of IgA FcRs [7] and in this way neutrophils show an increased ability to bind IgA. Literature data [10-12] postulated that FcRs play a crucial role in the activation and/or down-regulation of immune responses and thus, their ligation with pathogenic autoantibodies may have results in tissue damage, including blister formation in autoimmune blistering dermatoses [13]. The mechanism of inflammatory induction probably involves structural/conformation alternation of FcRs and modification of their activating/inhibitory functions. There are several known receptors for IgA, although few were characterized in much detail [2]. The best known IgA FcRs include the polymeric IgG recep-

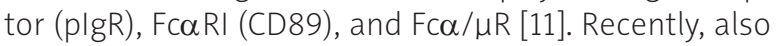
the role of CD71 in the pathomechanism of IgA-mediated diseases was noticed [14]. Still, a fascinating issue to be resolved is the identification of IgA FcRs involved in $\mathrm{DH}$ pathophysiology. To date, CD89 expressed on neutrophils, seems to be an essential receptor in $\mathrm{DH}[2,9]$.

It is known that $\mathrm{DH}$ is associated with gluten-sensitive enteropathy (GSE) and therefore may be called glutensensitive dermopathy [15], however the pathogenetic link between them still seems to be obscure. Interestingly, it is universally thought that $\mathrm{DH}$ may be a skin manifestation of celiac disease (CD) [16] and the connection between these disorders involves two different approaches (nosological and pathological). The development of $\mathrm{DH}$ as well as CD is a result of an immune response to gluten antigen, which is a substrate for transglutaminases (TGS). Nevertheless, the trigger/triggers of autoimmune response to TGs and factors inducing progression from $C D$ to $D H$ remain largely unclear [3].

There is no doubt that the term "dermatitis herpetiformis" is a misnomer for a specific form of autoimmune blistering dermatosis. Its name is a result of historical aspects. As mentioned above, the DH term was coined by Duhring in 1884. He proposed finding a place for a number of cases with nameless and misdiagnosed skin diseases, however no pathological verification was provided. The concept of DH was popularized over the years. Etymologically, the term "herpes" comes from the Greek and means "to crawl" [17]. However, nowadays it is mainly associated with the herpes viruses. Thus, this controversial name might imply a role of infection with the herpes viruses in the pathogenesis of $\mathrm{DH}$, which has never been proved to exist. Perhaps, the name reflects similar clinical pictures of both diseases (characteristic grouping of vesicles on erythematous base) [18], however it should be noted that the morphology of DH has been changing. Furthermore, there was no effective treatment of $\mathrm{DH}$ in Duhring's times and it is known that in untreated cases, the severity of disease may vary significantly during its course. Therefore, DH as now generally used is descriptive of the disease without any implication as to its pathophysiology. Thus, we propose that thorough consideration should be given to updating the definition of $\mathrm{DH}$ and, possibly, a new term in recognition of some important aspect of its cutaneous pathophysiology should be coined. Regarding this issue, it should be noticed that the older term "herpes gestationis" has already been replaced with the term "pemphigoid gestationis" as it better reflects the pathophysiology of this autoimmune blistering dermatosis. Perhaps then the term "gluten-sensitive IgA/neutrophil-mediated dermatitis" (the acronym GAND would sound nicely) for dermatosis described by Duhring would be more understandable for non-dermatologic medical/pharmaceutical/biotechnological community and lay public alike.

\section{Aim}

In light of the above we attempted to analyze whether in human DH there are any correlations between levels of serum IgA autoantibodies to eTG, tTG, $n p G$, as well as between levels of serum IgA autoantibodies to eTG, tTG, npG and the intensity of NE expression in lesional skin.

\section{Material and methods}

Altogether, $31 \mathrm{DH}$ patients showing cutaneous lesions active enough to prompt them to seek dermatological attention were examined. The clinical suspicion of DH was confirmed and thus DH diagnosis established with direct immunofluorescence showing any of 7 diagnostic patterns possible of microgranular/fibrillar IgA deposition and corroborated with histologic hematoxylin and eosin staining, $\mathrm{H}+\mathrm{E}$, performed in all cases. Patients were recruited during a period from January 2009 to July 2011 at the Histopathology and Immunopathology Section, Department of Dermatology, Poznan University of Medical Sciences, Poland. The serum used in the serological tests was taken at the time of hospital admission/ambulatory care. Five milliliters of blood serum were obtained from each individual and anti-eTG/anti-tTG/anti-npG IgA antibodies were evaluated with three commercially available ELISAs. The level of circulating serum IgA autoantibodies against the fusion protein containing nonapeptides of gliadin was evaluated with Anti-Gliadin (GAF-3X) ELISA (Euroimmun, Germany) with the manufacturer's cut-off $25 \mathrm{RU} / \mathrm{ml}$. The level of circulating serum IgA autoantibodies against tTG was detected with Anti-tTG ELISA (Euroimmun, Germany) with the manufacturer's cut-off $20 \mathrm{RU} / \mathrm{ml}$. The level of circulating serum IgA 
autoantibodies against eTG was detected with Anti-eTG ELISA (Immundiagnostic, Germany) with the manufacturer's cut-off $18 \mathrm{AU} / \mathrm{ml}$. All three tests are recommended by producers as useful in DH diagnosis. All measurements were made in the ELISA plate reader by a single operator following the manufacturer's instructions.

The intensity of NE expression in lesional skin were evaluated with immunohistochemical technique (IHC) on paraffin-embedded sections of lesional skin subjected to $4 \mu \mathrm{m}$ and then mounted on poly-L-lysine-coated glass slides. The immunohistochemical staining was performed with the use of a mouse monoclonal antibody to human NE (Dako, Denmark) and LSAB + system+ HRP visualization kit (Dako, Denmark). The primary antibody was diluted at $1: 100$. Then, slides were washed with PBS, counterstained with hematoxylin, coverslipped, and examined by light microscopy (BX40, Olympus, Japan) under magnification 200x and the area with visibly the most intense NE deposition was digitally photographed. The digital microscopic image analysis with quantitative digital morphometric software (Analyser 4D, [19]) was then used to
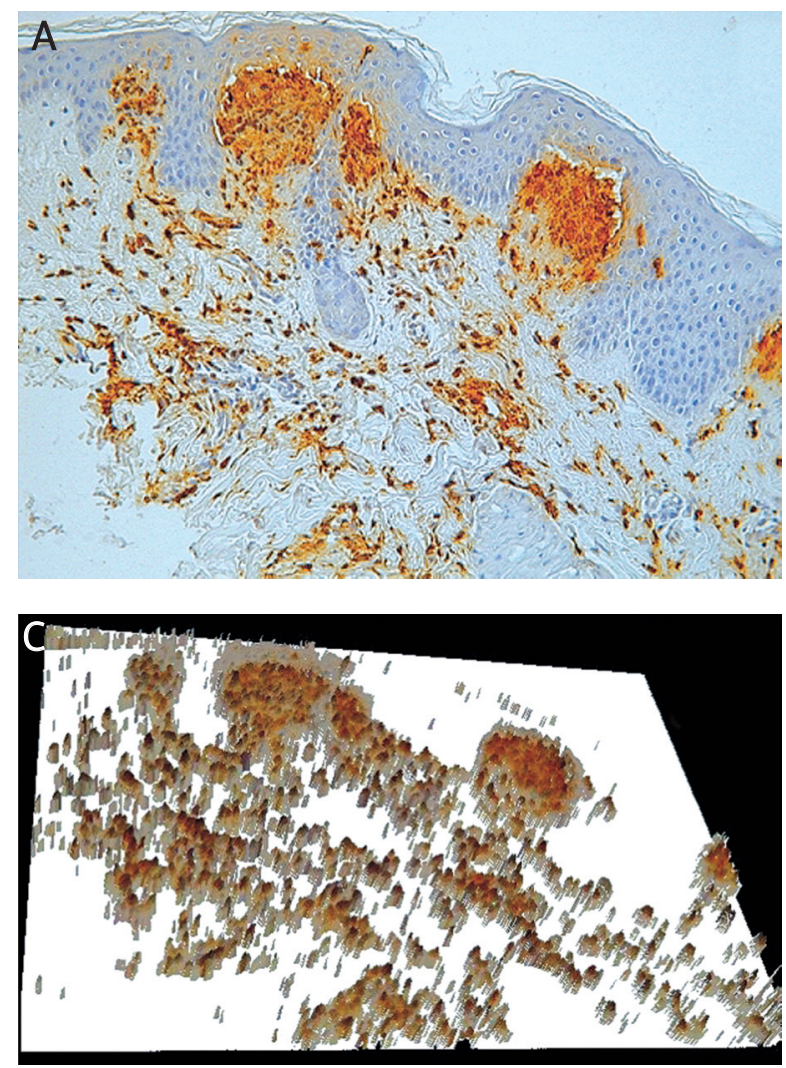

measure the intensity of positive immunostaining signals of the NE, i.e. NE expression, in percentages of expression [9].

\section{Statistical analysis}

Statistical analysis was done using Spearman's rank correlation coefficient to find out associations between the intensity of NE deposition and the levels of IgA autoantibodies to eTG, tTG, npG, as well as between the levels of IgA autoantibodies to eTG, tTG, npG in DH.

\section{Results}

Intensity of NE deposits processed with digital microscopic image analysis in a representative $\mathrm{DH}$ patient lesional skin is shown in Figure 1. The analysis of correlation showed insignificant correlations between the intensity of cutaneous NE expression and the levels of serum IgA antibodies to eTG, tTG, npG in patients with $\mathrm{DH}$. The following results were obtained: intensity of NE expression vs. anti-eTG IgA level $r=-0.1013$, intensity
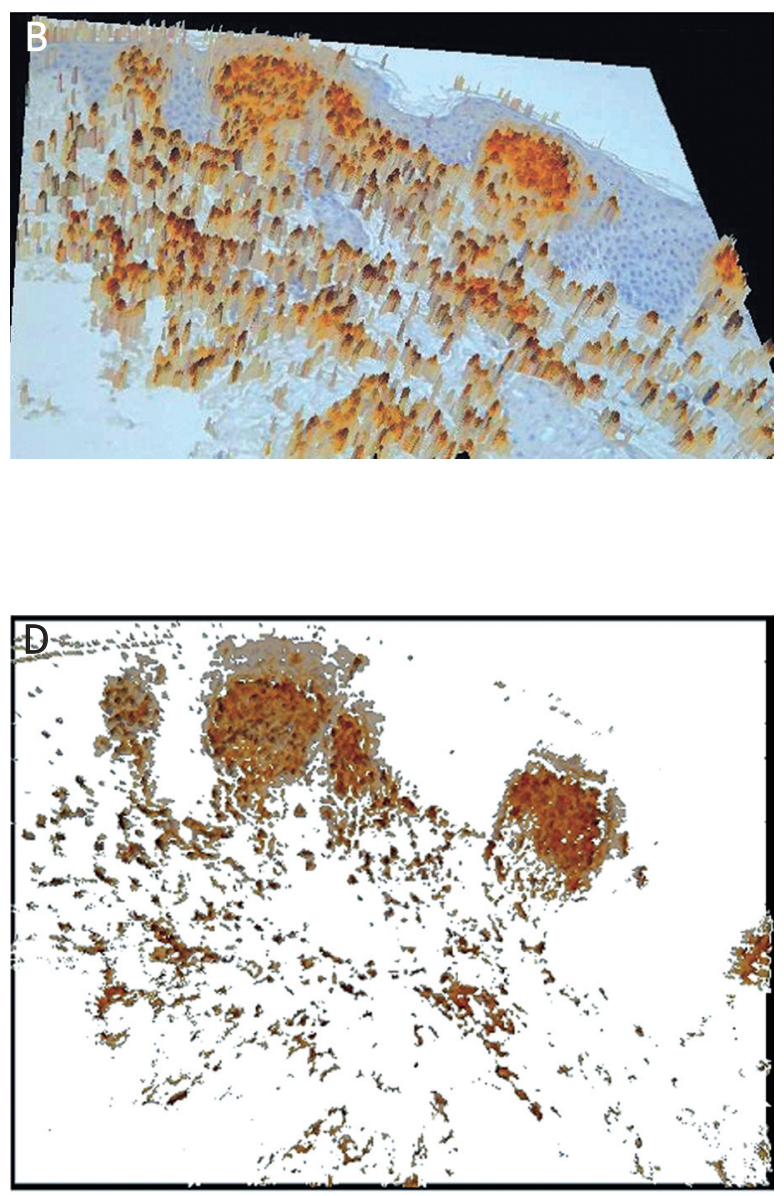

Figure 1. NE deposits in immunohistochemistry in lesional skin of a patient with $\mathrm{DH}$ (immunoperoxidase staining on paraffin embedded sections, original magnification 200x) (A). Intensity of NE deposits processed with digital microscopic image analysis superimposed on NE deposits immunohistochemistry: 3D (B). Intensity of NE deposits processed with digital microscopic image analysis: 3D (C). Intensity of NE deposits processed with digital microscopic image analysis: 2D (D) 

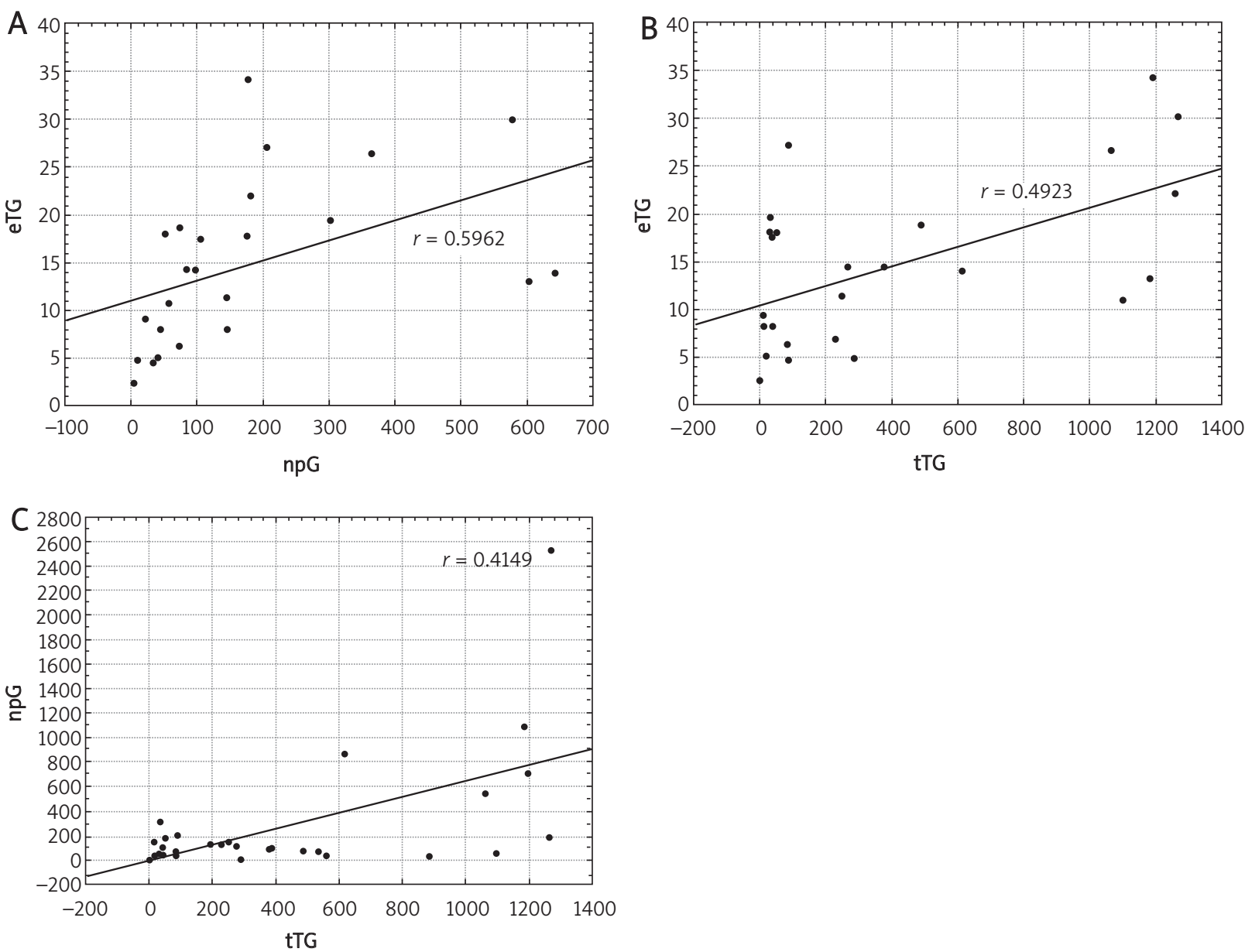

Figure 2. A significant correlation between: the level of serum IgA anti-eTG vs. anti-npG autoantibodies (A), the level of serum IgA anti-eTG vs. anti-tTG autoantibodies (B), the level of serum IgA anti-npG vs. anti-tTG autoantibodies (C) in $\mathrm{DH}$. The trend lines are shown

of NE expression vs. anti-tTG IgA level $r=-0.0505$, intensity of NE expression vs. anti-npG IgA level $r=-0.1766$.

There were statistically significant correlations ( $p<0.05$ ) between the levels of eTG, tTG, npG IgA autoantibodies. The following results were obtained: anti-eTG IgA vs. anti-tTG IgA $r=0.4923$, anti-eTG IgA vs. anti-npG IgA $r=0.5962$, anti-tTG IgA vs. anti-npG IgA $r=0.4149$. The results of correlations between eTG, tTG, npG IgA autoantibodies are shown in graphs in Figure 2.

\section{Discussion}

The plausible immunopathogenesis of $\mathrm{DH}$ involves activation of neutrophils by engaging their CD89 receptors with IgA (particularly IgA1) immunocomplexes (IC) $[2,9]$. This series of events leads to the release of NE, the proteolytic enzyme which degrades the DEJ and finally induces the blister formation [2, 9, 20]. The probable mechanism of action includes the shedding of cellular CD89, interaction with IgA and a change in IgA catabo- lism $[2,12]$. This scheme might lead to the NE releasing, DEJ destroying and consequently the intra-lamina lucida blister forming. However, there are no data clearly indicating this process as specific autoantibodies-dependent. Data obtained in this study have shown that there is no correlation between levels of circulating IgA autoantibodies to eTG, tTG, npG and the intensity of cutaneous $\mathrm{NE}$ expression in patients with $\mathrm{DH}$. This finding is in accordance with our previous data [9], where BowszycDmochowska et al. demonstrated just low strength of the correlation between NE expression and IgA to eTG in $\mathrm{DH}$. Thus, it seems that in human DH the activation of neutrophils is unrelated to the levels of serum IgA to eTG, tTG and npG. Based on these observations, we have hypothesized that in $\mathrm{DH}$ pathogenesis various local factors are probably more significant than pathogenetic association with GSE. Presumably, these local factors may involve FcRs on neutrophils, as the key mediators of IgA - and particularly important in the DH development IgA1 - effector function. However, the link between 
GSE and DH probably should be considered in light of the epitope spreading (ES) phenomenon. We suppose that these processes are plausibly responsible for manifestations of $\mathrm{DH}$.

It was reported that DH is associated with the HLAB8/DRw3 haplotype, which might be related with defective Fc receptor function in vivo [21]. So far, no functional study has been reported in association of FcRs with accumulation of pathogenic IgA1 in patients with $\mathrm{DH}$. Interestingly, examining GSE Lavö et al. [22] revealed that a relation between immunoglobulin (IgA) and FcRs mediated pathway may be considered in the impaired clearance of circulating ICS. This relation may happen via one pathway, which leads to the accumulation of ICs and subsequently to saturation of FcRs and decreased clearance via other pathway [22]. There are two hypotheses containing the causes of impaired clearance of circulating ICS in $\mathrm{DH}$ : it may be a result of (i) a primary defect in FCRs function with secondary accumulation of circulating ICs, (ii) saturation or hindrance to binding to the FcRs by circulating ICs [22]. Probably a different type of IgA FcRs may participate in sequential steps to promote $\mathrm{DH}$ lesions development. It seems that CD89 is a key receptor, which may be involved in the formation/amplification of the IgAIC. However, the function of other IgA FcRs should be investigated, e.g. a possible role of CD71. CD71 is involved in transcytosis of IgA in CD $[23,24]$ and probably implicated in its pathogenesis $[23,25]$. CD71 was recently identified as a new receptor for polymeric IgA [26], which shows affinity for IgA1, so it should be examined in $\mathrm{DH}$ as mediating cutaneous deposition of IgA1. Interestingly, CD71, but not CD89, participates in protected gliadin transport in $C D[23,24]$. Furthermore, it can be postulated that not only IgA isotype may participate in the process of binding to the FcRs during the induction of inflammation in $\mathrm{DH}$. It is known that human CD89 shares homology with receptors specific to the Fc region of IgG (FcyRs) and IgE (FcERIs) [27]. Thus, an important issue relates to whether it can promote inflammation on its own or must operate in tandem with another proinflammatory mediator (e.g. IgA-IgG or IgA-IgE). Moreover, it seems that the release of proinflammatory molecules, like NE, may happen in another way as through ICs. In light of this, the FcRs mutations, which affect the level of receptor affinity, can play a significant role in the DH immunopathogenesis [2]. There are data describing the FcRs polymorphism in BP with the effect on modulation of susceptibility to acquire this disease [28]. However, despite this being an interesting and essential issue for $\mathrm{DH}$ understanding as it seems, little is known about the IgA-FcRs polymorphisms and in vivo studies to investigate its functional importance in DH induction are still lacking.

It is known that ES occurs in cutaneous autoimmune diseases [29], however the functional studies of this phenomenon are scanty. According to Vanderlugt and Miller [30], ES may be defined as the development of immune response to endogenous epitopes secondary to the release of self-antigens during a chronic autoimmune or inflammatory response. Thus, the initial inflammatory process of DH might result in further DEJ destruction, which releases hidden or sequestered antigens, thereby inducing an autoimmune response against DEJ [31]. Ameen et al. [31] suggested that the targets of immune response do not remain fixed, but may be extended to include other epitopes on the same protein or other proteins in the same tissue. In the context of the above, $\mathrm{DH}$ has been described in association with $\mathrm{CD}$ and BP [32-34]. It can be postulated that cell-mediated immunity in GSE predisposes to ES with the development of subsequent and progressive cutaneous autoimmunity in $\mathrm{DH}$. It is assumed that CD patients have autoantibodies to tTG, DH patients to eTG and gluten ataxia patients to TG6 [32]. However, the question is whether these populations of autoantibodies are a result of ES or if they are truly directed towards tTG, eTG, TG6, is not clear [32]. Notably, in humans TTG and ETG are encoded on the same chromosome-20q11-12 [32]. Thus, cross reactivity and ES in the family of TGs would not be unexpected [35]. This is consistent with data of this study indicating that IgA to eTG/tTG/npG are produced in a coordinated way, what might correspond with suggested involvement of initially ES and subsequently cross reactivity in $\mathrm{DH}$ autoimmune response. Therefore, ES to TG3 may be responsible for the development of $\mathrm{DH}[5,36]$. On the other hand, current data [37] indicated that autoantibodies in most patients do not cross-react between different TGs isoforms.

It is observed that IgA1 is a predominated subclass in the sera of $\mathrm{DH}$ patients and IgA2 prevail in mucosal secretion of the colon [38]. Cutaneous IgA in DH consisted of both IgA1 and IgA2, although IgA1 predominated, however there are data about findings of IgAl alone in the $\mathrm{DH}$ skin [39]. A recent study has also shown the increased skin-homing potential of IgA1-plasmablast compared with IgA2 cells in $\mathrm{DH}$ [40], what may be related with previous papers that demonstrated IgA1 deposits in the skin of DH patients [41, 42]. Therefore, presented findings suggest that an increased skin-homing potential of IgA1 cells compared with IgA2 cells in DH could contribute to the formation of IgA1 deposits in the skin of DH patients. Interestingly, CD patients do not have IgA1, what may be evidence of selective entrapment of blood mononuclear cells to the DH skin [39]. Thus, plausibly IgA in the skin of $\mathrm{DH}$ patients is not produced in the gut or alternatively if it shows gut-mediated production, then only IgA1 is involved in the formation of cutaneous lesions [40], which might support our thesis of the importance of local factors in DH manifestation. Moreover, a local inflammatory process in the presence of IgA1 deposits at the DEJ would allow for the rolling and tethering of neutrophils to firmly adhere to vessel walls and move to the DEJ with resultant characteristic skin lesions [7]. 
Thus, it could be concluded from this study that in $\mathrm{DH}$ the engagement of either IgA to tTG, IgA to eTG or IgA to $n p G$, on cutaneous neutrophils might not be a principal stimulus to releasing NE. Still, it is possible that this stimulus is provided not by pooled IgA to tTG, eTG, npG, but specifically by IgA1 subclass [9]. This study revealed no connection between releasing of DEJ-degrading molecules and apparently pathogenic autoantibodies, so pathogenic anti-DEJ insult may arise following non-antibodies-mediated damage to the cutaneous DEJ. Strikingly, dapson, the drug of choice for cutaneous rash of $\mathrm{DH}$, does not seem to act as an antigen-antibody reaction suppressor. Likewise, iodides do not appear to exacerbate $\mathrm{DH}$ via pathways dependent on an antigen-antibody reaction. A young man with rheumatoid arthritis treated with methylprednisolone orally and azathioprine orally whose cutaneous itchy lesions, diagnosed as DH at the Histopathology and Immunopathology Section, Department of Dermatology, Poznan University of Medical Sciences, Poland, manifested itself despite immunosuppressive treatment might serve as an argument for the importance of non-autoimmune phenomena in cutaneous pathology of $\mathrm{DH}$. Moreover, our data support the notion that neutrophil activating capacities might be epitope-specific and plausibly dependent on changing autoantibody profiles and FcRs dysfunction.

\section{Conclusions}

It seems that in human DH lesional skin, the activation of neutrophils, judged by the NE expression, is unrelated to the levels of serum IgA autoantibodies to eTG/tTG/npG, which might imply the role of local factors in cutaneous inflammatory process in $\mathrm{DH}$. Apparently IgA autoantibodies to eTG/tTG/npG in DH are produced in a coordinated way, what might correspond with the proposed involvement of initially epitope spreading and subsequently cross reactivity in $\mathrm{DH}$ autoimmune response.

\section{Acknowledgments}

We wish to thank Euroimmun (Lübeck, Germany) for providing a part of commercial kits of Anti-tTG and AntiGliadin (GAF-3X) IgA ELISA used in this study.

This study was partly funded from the grant of the Polish Ministry of Science and Higher Education (N N 40100 1239 ).

Justyna Gornowicz-Porowska was supported by the Voivodeship Labour Office in Poznan (scholarship from project called "Scholarships for PhD students from faculties regarded as strategic for Wielkopolska region development", subsection 8.2.2. Operational Programme: Human Capital).

A part of this study was submitted for presentation at the $8^{\text {th }}$ International Congress on Autoimmunity (9-13 May 2012, Granada, Spain), and publication in the abstract form on the Congress website and Congress CD-ROM: Gornowicz-Porowska J, Bowszyc-Dmochowska M, Seraszek-Jaros A, Kaczmarek E, Dmochowski M. Human dermatitis herpetiformis: IgA autoantibodies to gliadin nonapeptides, tissue and epidermal transglutaminases are linked, but unrelated to cutaneous neutrophil elastase.

\section{References}

1. Duhring LA. Dermatitis herpetiformis. JAMA 1884; III: 225-9. 2. Gornowicz-Porowska J, Bowszyc-Dmochowska M, Dmochowski M. Autoimmunity-driven enzymatic remodeling of the dermal-epidermal junction in bullous pemphigoid and dermatitis herpetiformis. Autoimmunity 2011 Sep 14 Epub ahead of print, PMID: 21916544.

3. Gornowicz-Porowska J, Bowszyc-Dmochowska M, SeraszekJaros A, et al. Association between levels of IgA antibodies to tissue transglutaminase and gliadin-related nonapeptides in dermatitis herpetiformis. TSWJ 2011 in press.

4. Dieterich W, Laag E, Bruckner-Tuderman L, et al. Antibodies to tissue transglutaminase as serologic markers in patients with dermatitis herpetiformis. J Invest Dermatol 1999; 113: 133-6.

5. Sárdy M, Kárpáti S, Merkl B, et al. Epidermal transglutaminase (TGase 3 ) is the autoantigen of dermatitis herpetiformis. J Exp Med 2002; 195: 747-57.

6. Schwertz E, Kahlenberg F, Sack U, et al. Serologic assay based on gliadin-related nonapeptides as a highly sensitive and specific diagnostic aid in celiac disease. Clin Chem 2004; 50: 2370-5.

7. Hall RP, Takeuchi F, Benbenisty KM, Streilein RD. Cutaneous endothelial cell activation in normal skin of patients with dermatitis herpetiformis associated with increased serum levels of IL-8, sE-selectin, and TNF-alpha. J Invest Dermatol 2006; 126: 1331-7.

8. Smith AD, Streilein RD, Hall RP. Neutrophol CD11b, L-selectin and FC IgA receptors in patients with dermatitis herpetiformis. Br J Dermatol 2002; 147: 1109-17.

9. Bowszyc-Dmochowska M, Seraszek A, Kaczmarek E, et al. Low strength of correlation between the intensity of neutrophil elastase expression in lesional skin and the level of serum IgA antibodies to epidermal transglutaminase in dermatitis herpetiformis. The Open Autoimmunity Journal 2009; 1: 1-4.

10. Nimmerjahn F, Ravetch JV. Fgammac receptors as regulators of immune response. Nat Rev Immunol 2008; 8: 34-47.

11. Gomes M, Herr AB. IgA and IgA-specific receptors in human disease: structural and functional insights into pathogenesis and therapeutic potential. Springer Semin Immunopatol 2006; 28: 383-95.

12. Wines BD, Hogarth PM. IgA receptors in health and disease. Tissue Antigens 2006; 6: 103-14.

13. Zhao M, Trimbeger ME, Li N, et al. Role of FCRs in animal model of autoimmune bullous pemphigoid. J Immunol 2006; 177: 3398-405.

14. Haddad E, Moura IC, Arcos-Fajardo M, et al. Enhanced expression of the CD71 mesangial IgA1 receptor in Berger Disease and Henoch-Schönlein Nephritis: association between CD71 expression and IgA deposits. J Am Soc Nephrol 2003; 14: 327-37.

15. Kárpáti S. An exception within the group of autoimmune blistering diseases: dermatitis herpetiformis, the gluten-sensitive dermopathy. Dermatol Clin 2011; 29: 463-8. 
16. Zone JJ. Skin manifestations of celiac disease. NIH Consensus Development Conference on Celiac Disease, June 28-30, 2004, Bethesda, Maryland, 73-75.

17. Ganczew B. Terminologia dermatovenerologica. Państwowy Zakład Wydawnictw Lekarskich, Warszawa 1972; 135.

18. Fry L. Derrmatitis herpetiformis. In: Management of blistering diseases. Wojnarowska F, Briggaman RA (ed.). Raven Press, New York 1990; 139-60.

19. Kaczmarek E, Strzelczyk R. From two to three-dimensional visualization of structures in light and confocal microscopy - applications for biomedical studies. In: Current issues on multidisciplinary microscopy research and education. Mendez Vilas A, Labajos-Broncano L (ed.). Badajoz: FORMATEX 2005; 289-95.

20. Oikarinen Al, Zone JJ, Ahmed AR, et al. Demonstration of collagenase and elastase activities in the blister fluids from bullous skin diseases. Comparison between dermatitis herpetiformis and bullous pemphigoid. J Invest Dermatol 1983; 81: 261-6.

21. Lawley TJ, Hall RP, Fauci AS, et al. Defective Fc-receptor functions associated with the HLA-B8/DRw3 haplotype. N Engl J Med 1981; 304: 185-92.

22. Lavö B, Nilsson B, Lööf L, et al. Fc receptor function and circulating immune complexes in gluten sensitive enteropathy - possible significance of serum IgA. Gut 1991; 32: 876-80.

23. Matysiak-Budnik T, Moura IC, Arcos-Fajardo M, et al. Secretory IgA mediates retrotranscytosis of intact gliadin peptides via the transferrin receptor in celiac disease. J Exp Med 2008; 205: 143-54

24. Meresse B, Ripoche J, Heyman M, Cerf-Bensussan N. Celiac disease: from oral tolerance to intestinal inflammation, autoimmunity and lymphomagenesis. Mucosal Immunology 2009; 2: 8-23.

25. Heyman M, Menard S. Pathways of gliadin transport in celiac disease. Ann N Y Acad Sci 2009; 1165: 274-8.

26. Moura IC, Centelles MN, Arcos-Fajardo M, et al. Identification of the transferrin receptor as a novel immunoglobulin (Ig)A1 receptor and its enhanced expression on mesangial cells in IgA nephropathy. J Exp Med 2001; 194: 417-25.

27. Woof JM. The human IgA-Fc alpha receptor interaction and its blockade by streptococcal IgA-binding proteins. Biochem Soc Trans 2002; 30: 491-4.

28. Guilabert A, Lozano F, Iranzo P, et al. The role of Fc gamma receptors polymorphisms in bullous pemphigoid. J Am Acad Dermatol 2010; 63: 161-3.

29. Chan LS, Vanderlugt CJ, Hashimoto T, et al. Epitope spreading lessons from autoimmune skin diseases. J Invest Dermatol 1998; 110: 103-19.

30. Vanderlugt CJ, Miller SD. Epitope spreading. Current Opin Immunol 1996; 8: 831-6.

31. Ameen M, Bhogal BS, Black MM. Dermatitis herpetiformis evolving into bullous pemphigoid: a probable example of epitope spreading. Clin Exp Dermatol 2000; 25: 398-400.

32. Stamnaes J, Dorum S, Fleckenstein B, et al. Gluten T cell epitope targeting by TG3 and TG6: implications for dermatitis herpetiformis and gluten ataxia. Amino Acids 2010; 39: 1183-91.

33. Setterfield J, Bhogal B, Black MM, McGibbon DH. Dermatitis herpetiformis and bullous pemphigoid: a developing association confirmed by immunoelectronmicroscopy. Br I Dermatol 1997; 136: 253-6.

34. Sander HM, Uty MMP, Peters MS. Bullous pemphigoid and dermatitis herpetiformis: mixed bullous disease or coexistence of two separate entities? J Cutan Pathol 1989; 16: 370-4.
35. Grenard P, Bates MK, Aeschlimann D. Evolution of transglutaminase genes: identification of a transglutaminase gene cluster on human chromosome 15q15. J Biol Chem 2001; 276: 33066-78.

36. Zone JJ, Schmidt LA, Taylor TB, et al. Dermatitis herpetiformis sera or goat anti-transglutaminase-3 transferred to human skin-grafted mice mimics dermatitis herpetiformis immunopathology. J Immunol 2011; 186: 4474-80.

37. Hadjivassiliou M, Aeschlimann P, Strigun A, et al. Autoantibodies in gluten ataxia recognize a novel neuronal transglutaminase. Ann Neurol 2008; 64: 332-43.

38. Dmochowski M. Opryszczkowate zapalenie skóry. In: Autoimmunizacyjne dermatozy pęcherzowe. Dmochowski M (ed.). Wydawnictwo Naukowe Akademii Medycznej im. Karola Marcinkowskiego w Poznaniu, Poznań 2006; 281-311.

39. Hall RP, Lawlez TJ. Characterization of circulating and cutaneous IgA immune complexes in patients with dermatitis herpetiformis. J Immunol 1985; 135: 1760-5.

40. Kantele JM, Savilahti E, Westerholm-Ormio M, et al. Decreased numbers of circulating plasmablasts and differences in IgA1-plasmablast homing to skin in celiac disease and dermatitis herpetiformis. Clin Exp Immunol 2009; 156: 535-41.

41. Olbricht SM, Flotte TJ, Collins B, et al. Dermatitis herpetiformis. Cutaneous deposition of polyclonal IgA1. Arch Dermatol 1986; 122: 418-21.

42. Fry L. Dermatitis herpetiformis. Baillières Clin Gastroenterol 1995; 9: 371-93. 